\title{
ANÁLISE DA RELAÇÃo ENTRE A PRÁTICA DO LEAN E O DESEMPENHO DE PRAZO DE PROJETOS EM ORGANIZAÇÕES REPRESENTATIVAS DE PESQUISA CLÍNICA
}

\author{
Camila Maldonado (maldonado_camila@ hotmail.com) - Fundação Vanzolini \\ Ana Paula Lopes (paulavlopes@ outlook.com) - Engenharia de Produção - FEI \\ Marly Monteiro de Carvalho (marlymc@usp.br) - Engenharia de Produção - POLI USP
}

\section{RESUMO}

Nos tempos atuais, as empresas buscam respostas rápidas e eficazes que visam aproveitar oportunidades com melhor controle e uso dos recursos existentes. Tradicionalmente, metas de prazo são utilizadas como critérios para medição de sucesso de projetos. $O$ investimento na adoção de técnicas e ferramentas de gerenciamento pode contribuir de maneira significativa para o sucesso do projeto. A aparição das histórias de sucesso associada à adoção de ferramentas de melhoria contínua está convergindo para encorajar os líderes a considerar o lean em suas instituições e empresas. A metodologia lean é reconhecida pela maximização do valor por meio da contínua eliminação de desperdícios e foi originalmente concebida como uma prática de manufatura, porém a mentalidade tem sido gradualmente disseminada em todas as áreas empresariais e também para empresas dos mais diferentes tipos e setores, tornandose efetivamente uma filosofia e uma cultura empresarial. Nas últimas décadas, muitos estudos foram publicados na tentativa de entender a relação da filosofia lean como estratégia de sobrevivência que garanta ganhos na produtividade e vantagens competitivas, porém ainda é um fenômeno experimentado por um seleto grupo de Organizações Representativas de Pesquisa Clínica (ORPC). Na tentativa de contribuir com esses estudos, esta pesquisa busca analisar as relações entre o uso da metodologia lean e o desempenho de prazo de projetos em ORPCs através de uma abordagem qualitativa de estudo de caso. A conclusão da pesquisa indica que a inclusão dos princípios lean como parte da missão, cultura e valores da companhia, bem como o uso de ferramentas de melhoria contínua podem contribuir com a entrega antecipada das atividades dos projetos e facilitar a obtenção dos objetivos estratégicos da empresa.

Palavras-chave: Gerenciamento de Projetos, Gerenciamento de Prazos, Desempenho de Projetos, Metodologia Lean, Organização Representativas de Pesquisa Clínica.

Área: Gestão de projetos de desenvolvimento de produtos e serviços.

\section{INTRODUÇÃO}

De acordo com (PMI, 2013), um projeto é um esforço temporário empreendido para criar um produto, serviço ou resultado exclusivo. Os processos de gerenciamento de projetos são agrupados em áreas de conhecimento distintas de acordo com o guia. Cada área representa um conjunto completo de conceitos, termos e atividades que compõem um campo profissional, campo de gerenciamento de projetos ou uma área de especialização. 
O sistema de gestão da qualidade em projetos, uma das áreas de conhecimento do PMBOK®, é de extrema importância para as empresas que procuram instrumentos que garantam satisfação de seus clientes, melhoria contínua de seus processos e produtos e, consequentemente, a obtenção de bons resultados.

Neste contexto, muitas empresam adotaram a filosofia lean como estratégia para aumentar a satisfação dos clientes por meio da melhor utilização dos recursos em todos os níveis da organização. Lean é a busca pela maximização do valor por meio da contínua eliminação de desperdícios e foi originalmente concebida como práticas de manufatura, porém a mentalidade tem sido gradualmente disseminada em todas as áreas empresariais e também para empresas dos mais diferentes tipos e setores, tornando-se efetivamente uma filosofia e uma cultura empresarial (LEAN INSTITUTE BRASIL, 2016).

Para Teich e Faddoul (2013, p.75), lean na área da saúde pode ser definido como "um compromisso cultural de uma organização de aplicar o método científico ao projeto, ao desempenho e, continuamente, à melhoria do trabalho feito por equipes de pessoas, levando a, mensuravelmente, melhor valor para pacientes e outras partes interessadas".

O principal objetivo desta pesquisa é analisar a relação entre o uso da metodologia lean e o desempenho de prazo de projetos em Organizações Representativas de Pesquisa Clínicas (ORPC), cuja principal atividade é conduzir projetos de pesquisa clínica em nome dos seus clientes farmacêuticos com a finalidade de agilizar o processo de desenvolvimento e aprovação de medicamentos bem como dispositivos médicos.

A estrutura desta pesquisa foi dividida em cinco capítulos para encadear de forma lógica a pesquisa proposta. O primeiro capítulo é introdutório de maneira a contextualizar os principais tópicos abordados no trabalho. No segundo capítulo, encontramos a revisão da literatura, o enquadramento histórico, princípios e ferramentas da filosofia lean. Neste capítulo encontramos ainda conceitos relacionados ao desempenho de projetos e também considerações da influência da metodologia lean no desempenho de projetos.

No terceiro capítulo é descrito o método de pesquisa utilizada e instrumentos de coleta de dados e o quarto capítulo mostra os resultados obtidos. No quinto e último capítulo está consumada uma síntese do trabalho produzido, ideias-chave, as principais conclusões, correlacionando o método de pesquisa com os objetivos de pesquisa e possíveis aspectos de desenvolvimento futuro.

\section{REVISÃO TEÓRICA}

Este capítulo tem como objetivo apresentar a revisão teórica por meio da busca e seleção na literatura dos principais autores e conceitos relacionados ao objeto de estudo.

\subsection{Metodologia Lean}

O termo Lean Production está intimamente ligado ao Toyota Production System (TPS) ou Sistema Toyota de Produção e refere-se a uma filosofia de administração da produção que surgiu no Japão na década de 50 a partir do trabalho dos engenheiros Taiichi Ohno e Eiji Toyoda da Toyota Motor Company que, ao visitarem fábrica da Ford Motor nos Estados Unidos, até então a unidade de manufatura mais eficiente do mundo com o sistema de produção em massa, desenvolveram um sistema de produção que poderia ser aplicado nas empresas japonesas no período pós Segunda Guerra Mundial, onde a insuficiência de recursos humanos, financeiros e materiais eram incontestáveis (WOMACK; JONES; ROOS, 1992). 
Com isso, as empresas estão buscando muitos programas de melhoria contínua (ZU; ROBBINS; FREDENDALL, 2010). O Project Management Institute (Instituto de Gerenciamento de Projetos) aponta que os programas de melhoria contínua reduzem o desperdício e eliminam as atividades que não agregam valor, permitindo que os processos sejam realizados com níveis mais elevados de eficiência e eficácia.

Womack e Jones (1998) estabelecem um conjunto de cinco princípios fundamentais e orientadores do Lean Thinking: criação de valor, fluxo de valor, fluxo, puxar e perfeição. Ainda de acordo com a mesma pesquisa, o primeiro princípio - criação de valor - é essencial no pensamento enxuto e consiste em identificar produtos e serviços que satisfaçam as necessidades dos clientes a um preço específico e em um momento específico. É o cliente final que define o que é valor, portanto oferecer o produto ou serviço errado da forma correta é desperdício.

\subsubsection{Ferramentas}

Ao longo da evolução do Toyota Production System, muitas ferramentas ou métodos foram desenvolvidos para ajudar a eliminar o desperdício (ZIDEL, 2006) e sustentar a filosofia lean. Enumerá-los todos sairia do âmbito desta monografia, porém abaixo são listadas algumas ferramentas que ajudam uma organização no movimento em direção a uma transformação lean. São elas: conceito 5S, trabalho padronizado, gestão visual, TPM (Total Productive Maintenance ou Manutenção da Produtividade Total), kaizen e 5W.

$\mathrm{O}$ conceito de $5 \mathrm{~S}$ possui como base as cinco palavras japonesas cujas iniciais formam o nome do programa: Seiri (classificar), Seiton (organizar), Seiso (limpar), Seiketsu (padronizar), Shitsuke (disciplinar). É uma ferramenta que visa organização do espaço de trabalho através da disciplina, consciência e responsabilidade de todos os funcionários para tornar o local de trabalho organizado, seguro e produtivo (MICHALSKA; SZEWIECZEK, 2007).

A ferramenta TPM (Total Productive Maintenance ou Manutenção da Produtividade Total) visa aumentar a disponibilidade e a eficácia do equipamento existente, através de melhoria e manutenção dos equipamentos ao nível ideal, de forma a reduzir o seu custo de ciclo de vida e o investimento em recursos humanos (CHAN et al, 2005).

A técnica Kaizen (palavra japonesa para melhoria) corresponde a uma ferramenta bastante potente, já que gera a necessidade de melhorar de forma incremental e contínua todos os processos, através da participação dos funcionários (BRUNET; NEW, 2003). Segundo o kaizen, é sempre possível fazer melhor, nenhum dia deve passar sem que alguma melhoria tenha sido implantada, seja ela na estrutura da empresa ou no indivíduo. Cada pequena mudança empreendida por cada um, quando somadas ao longo do tempo trarão grandes resultados, sem perturbar o equilíbrio da estrutura.

A técnica dos " $5 \mathrm{~W}$ ou 5 Porquês" possui como base a palavras inglesa Why (porquê) e é uma técnica amplamente utilizada para encontrar a causa raiz de um defeito, desperdício ou problema. É uma técnica de análise que parte da premissa que após perguntar cinco vezes o porquê um problema está acontecendo, é possível determinar a causa raiz do problema ao invés da fonte de problemas. Quando a causa raiz é identificada, pode-se estabelecer a melhor maneira para correção (ZIDEL, 2006).

\subsection{Desempenho de Projetos}

A eficácia do processo de gerenciamento de projetos define se os projetos exercem grande influência na obtenção de vantagem competitiva para uma organização (BARBER, 
2004). Para Oisen (1971), o gerenciamento de projetos é um conjunto de ferramentas e técnicas que direcionam o uso de diversos recursos para realização de uma tarefa única, complexa e exclusiva, respeitando as exigências de tempo, custo e qualidade; definição esta que inclui alguns critérios de sucesso, conhecido como triângulo de ferro.

Segundo Patah e Carvalho (2012, p. 200), "há indícios que quanto maior o grau de implementação das metodologias de gestão de projetos, maior a chance de o projeto cumprir seu prazo planejado".

Atkinson (1999) relata que embora algumas pesquisas tragam diferentes sugestões para definir gerenciamento de projetos, os critérios de sucesso nomeados como custo, prazo e qualidade permanecem presentes nas definições atuais. Para Berssaneti (2011), o desempenho dos projetos é avaliado de acordo com o atendimento ao orçamento planejado, cronograma, especificações técnicas, e atendimento às demandas dos clientes.

O tempo é uma das variáveis mais imponderáveis e inflexíveis da gestão de projetos. É uma tarefa difícil quantificar de maneira precisa o custo do atraso de projetos, mas é certo que o custo existe e que atrasos geram insatisfação dos clientes e demais stakeholders, podendo ainda resultar na perda de uma oportunidade ou até de determinado seguimento de mercado (ROCHA et al., 2014).

Usualmente as metas de prazo, custo e qualidade são utilizadas como base para medição do sucesso de um projeto (BERSSANETI, 2011). Com isso, um projeto que não se distanciasse excessivamente do orçamento planejado, cumprisse o cronograma e os requisitos estabelecidos pelos stakeholders, seria considerado um projeto de sucesso.

Segundo Ling (2004), não apenas as métricas de tempo e qualidade como também as métricas de atendimento da satisfação do cliente podem ser utilizadas para avaliar o desempenho dos projetos. Para que seja possível medir o sucesso em projetos, é possível estabelecer critérios e métricas. Ainda de acordo com a mesma pesquisa, as métricas mais utilizadas para medição de desempenho em projetos são aquelas relacionadas à obtenção dos valores inicialmente planejados de prazo e custo.

\section{MÉTODO DE PESQUISA}

O objetivo desta pesquisa é analisar a relação entre o uso da metodologia lean e o desempenho de prazo de projetos em Organizações Representativas de Pesquisa Clínicas (ORPC), cuja principal atividade é conduzir projetos de pesquisa clínica em nome dos seus clientes farmacêuticos com a finalidade de agilizar o processo de desenvolvimento e aprovação de medicamentos bem como dispositivos médicos.

O método de pesquisa adotado para este trabalho foi o estudo de caso com abordagem qualitativa. A empresa estudada é uma multinacional no ramo de life sciences que conduz ensaios clínicos em nome dos seus clientes farmacêuticos com a finalidade de agilizar o processo de desenvolvimento e aprovação de medicamentos. Atuante no segmento de pesquisa clínica há algumas décadas, a empresa é considerada uma organização representativa de pesquisa clínica de grande porte, presente em 51 países e com cerca de 19.000 funcionários, além de contar com uma carteira de clientes consolidada e marca reconhecidamente forte no mercado em que atua, com colaboradores experientes no desenvolvimento de soluções e condução de estudos clínicos de Fase I a IV. 
A coleta de dados envolveu entrevistas semiestruturadas, informações da web site da empresa e documentos complementares. Os entrevistados autorizaram a divulgação do conteúdo.

O roteiro das entrevistas foi dividido, basicamente, em duas seções. A primeira seção visa obter informações do indivíduo como principais funções e responsabilidades na empresa. A segunda seção visa compreender as questões referentes ao gerenciamento de projetos na ORPC, a participação dos colaboradores na aplicação da metodologia lean na empresa, as métricas de desempenho de prazos em projetos utilizadas e a influência do lean do desempenho de prazos.

Para a coleta de informações, foram feitas quatro entrevistas com funcionários da empresa (dois gerentes, um coordenador e um especialista) com atuação nos projetos de estudos clínicos a nível global. As entrevistas foram baseadas no roteiro semiestruturado, o qual foi elaborado com base na revisão teórica.

\section{RESULTADOS}

Os entrevistados possuem em média 10 anos de experiência no seguimento de pesquisa clínica e estão há mais de dois anos de empresa, o que demonstra e pode evidenciar o conhecimento que possuem da missão, cultura e valores da companhia bem como dos processos vigentes da empresa. Informações adicionais dos participantes deste estudo são apresentadas na Tabela 1.

Tabela 1. Características dos entrevistados

\begin{tabular}{cccc}
\hline Entrevistado & Nível & País de Trabalho & Abrangência de Projetos \\
\hline 1 & Gerente & Brasil & Global \\
2 & Gerente & Brasil & Global \\
3 & Coordenador & Brasil & Global \\
4 & Especialista & Brasil & Global \\
\hline
\end{tabular}

Como previsto na parte de metodologia, as entrevistas foram gravadas, transcritas e validadas com os entrevistados. As entrevistas foram conduzidas através do roteiro de pesquisa (ver Apêndice II) que foi intencionalmente dividido em seis blocos de perguntas para facilitar a avaliação dos dados e contribuir mais detalhadamente ao entendimento do fenômeno a ser estudado, conforme mostra a Tabela 2. Os dados obtidos foram relacionados à teoria pesquisada através de uma análise qualitativa que buscou verificar a percepção dos entrevistados em relação ao uso da metodologia lean e sua relação no desempenho de prazo de projetos em Organizações Representativas de Pesquisa Clínicas.

Tabela 2. Contextualização do Roteiro de Pesquisa 


\begin{tabular}{cl}
\hline Bloco de Perguntas & \multicolumn{1}{c}{ Abordagens Principais } \\
\hline 1 & Aplicação e disseminação da metodologia lean; princípios lean . \\
2 & Ferramentas lean : usos e aplicação. \\
3 & Treinamento dos colaboradores na metodologia lean. \\
4 & $\begin{array}{l}\text { Métricas de desempenho disponiveis. } \\
\text { Planejamento das atividades do projeto; uso de metodologias de boas } \\
\text { práticas. } \\
5\end{array}$ \\
6 & $\begin{array}{l}\text { Monitoramento e controle da evolução de desvios e situações de não } \\
\text { conforme. }\end{array}$ \\
\hline
\end{tabular}

O primeiro aspecto mencionado entre todos os entrevistados expõe que a mentalidade enxuta foi introduzida na empresa como um sistema de negócios a fim de obter resultados significativos e de maneira mais intensa, já que se acredita que o lean proporciona qualidade superior e a entrega de serviço aos clientes com o menor custo possível através da maximização de ações que acrescentam valor nas diferentes unidades de negócio, afirmação esta que vai de encontro com o que cita a literatura nos trabalhos de Khadem, Ali e Seifoddini (2008).

Todos os entrevistados também relataram que a empresa está iniciando uma transformação em relação ao movimento lean e como maneira de disseminar a metodologia, pode-se identificar os investimentos em treinamentos e a capacitação dos funcionários de todos os níveis hierárquicos no uso das ferramentas que sustentam a prática lean.

De acordo com Zidel (2016), para alcançar uma transformação lean, é essencial compreender completamente os princípios e ferramentas, bem como sua aplicação. Na percepção dos entrevistados, os princípios que norteiam toda a filosofia e que são mais facilmente identificados na corporação foram: a perfeição, fluxo de valor, criação de valor e fluxo contínuo. Estes quatro princípios fazem parte, juntamente com o sistema pull, do conjunto de cinco princípios descritos por Womack e Jones (1998) e orientam toda a filosofia lean.

Todos os entrevistados entendem que o maior desafio na utilização da metodologia lean é a disseminação linear dos princípios a todos os funcionários da empresa. $\mathrm{O}$ entrevistado 4 entende que o tempo é uma variável crucial já que os funcionários não têm tempo suficiente para estudar e aprender sobre a metodologia devido à grande carga de trabalho.

[...] é necessário absorver o que é transmitido sobre a metodologia e não simplesmente participar dos treinamentos sem que resulte em aplicações dos princípios e ferramentas rotineiramente. (Entrevistado 4)

No segundo bloco de perguntas que seriam sobre os usos e aplicações das ferramentas lean, todos os entrevistados, assim como Zidel (2006), entendem que diversas ferramentas são utilizadas rotineiramente durante a execução das atividades dos projetos para auxiliar na diminuição de desperdícios. Diversas ferramentas foram citadas pelos entrevistados, porém as mais comumente utilizadas e mencionadas são: $5 \mathrm{~W}$ ou 5 Porquês, Kaizen, trabalho padronizado, gestão visual e definição do problema (Why, Where, When, Who, What, How).

A maior parte das ferramentas mencionadas pelos colaboradores estão incorporadas aos procedimentos operacionais padrão a nível global, como forma de garantir que as tarefas sejam executadas de maneira padronizada nos diversos países onde um mesmo projeto de estudo 
clínico esteja acontecendo. $\mathrm{O}$ entrevistado 3 informou adicionalmente que a inclusão das ferramentas lean aos procedimentos operacionais padrão torna o seu uso como mandatório durante a execução de determinada tarefa.

Todos os entrevistados têm a percepção que a utilização de ferramentas pode auxiliar na entrega antecipada das atividades dos projetos e facilitar a obtenção dos objetivos estratégicos da empresa. Semelhantemente, Kerzner (2010) apresentou que a correta aplicação do conhecimento, processo, habilidades, ferramentas e técnicas pode ter um impacto significativo no sucesso do projeto.

No terceiro bloco de perguntas que seriam sobre o treinamento dos colaboradores na metodologia lean, foi exposto por $100 \%$ dos entrevistados que são fornecidos treinamentos aos funcionários e focados na compreensão dos conceitos básicos da metodologia lean, conceitos e aplicações das principais ferramentas, entendimento e identificação de desperdícios, identificação de oportunidades para o lean, entre outros.

Com relação ao monitoramento dos funcionários quanto às práticas e utilização de ferramentas lean para garantir a efetividade das entregas, os entrevistados 3 e 4 entendem que este monitoramento que está sendo gradualmente aprimorado. $\mathrm{O}$ entrevistado 3 entende que empresa está incorporando os princípios e ferramentas da filosofia enxuta nos principais sistemas computadorizados utilizados pelos funcionários durante a condução dos projetos. Os entrevistados 2 e 3 também acrescentam que o monitoramento ocorre através do coaching fornecido pelos gerentes diretos; afirmação esta que vai de encontro à pesquisa de Toussaint e Berry (2013) que diz que o lean é uma abordagem de gestão inovadora que transforma a cultura organizacional de dentro para fora, onde líderes devem tornar-se facilitadores e mentores, bem como permitir que todos os colaboradores participem diretamente das ações de melhoria. Apenas o entrevistado 1 entende que não há um monitoramento regular e constante quanto às práticas e utilização de ferramentas e sua relação direta com o sucesso de projetos.

Partindo para o quarto bloco de perguntas que se refere às métricas de desempenho disponíveis na empresa, os quatro entrevistados foram capazes de fornecer facilmente as métricas de desempenho disponíveis na empresa, bem com as utilizadas no departamento em que trabalham. São inúmeras as métricas disponíveis na empresa: pesquisa de satisfação dos clientes, pesquisas de satisfação dos funcionários, métricas de desempenho de processo, métricas financeiras, métricas de auditorias internas e externas, métricas de qualidade, indicadores de estratégia corporativa, planos estratégicos de departamentos. Segundo Ling (2004), é necessário estabelecer critérios e métricas para que seja possível medir o sucesso em projetos. Ainda, para Patah e Carvalho (2012), as métricas trazem consistência à gestão de projetos e permitem que decisões importantes possam ser tomadas com base em informações precisas.

No quinto bloco de perguntas, foram analisados os dados sobre o planejamento das atividades do projeto e o uso de metodologias de boas práticas. De acordo com o artigo de Ciriello (2011), para que os projetos tenham chances maiores de sucesso, o planejamento das atividades deve ser detalhado e finalizado antes da execução propriamente dita. Esta mesma pesquisa, traz que a utilização de metodologias de boas práticas é imprescindível para se atingir um índice satisfatório no cumprimento dos prazos no projeto. Adicionalmente, neste quinto bloco de perguntas foi questionado aos entrevistados se eles costumam fazem uma análise de desempenho visando verificar se a contribuição individual atendeu aos resultados desejados. Os quatros participantes relataram que a análise não é feita após a entrega de cada uma das 
atividades executadas do projeto. Em contrapartida, na literatura pesquisada, a medição de desempenho aparece como um indicador poderoso para alcançar os objetivos de melhoria contínua (ATTADIA; MARTINS, 2013). Adicionalmente, Neely (1998, p.5) ainda destacou que "um sistema de medição de desempenho permite que as decisões e ações sejam tomadas com base em informações porque ele quantifica a eficiência e a eficácia das ações passadas por meio da coleta, exame, classificação, análise, interpretação e disseminação dos dados adequados".

Referente ao último bloco de perguntas, o de monitoramento e controle da evolução de desvios e situações de não conforme, a literatura diz que é essencial na gestão de projetos o monitoramento e controle da evolução e que toda a equipe do projeto deve se empenhar e contribuir para que todas as atividades deste ciclo sejam executadas com perfeição (SILVA, 2016). Todos os entrevistados, referente a este tema, colocaram que conseguem identificar situações de não conforme e desvios de maneira imediata e com certa facilidade. Os quatro entrevistados também informaram que assim que os casos de não conforme e desvios são identificados, a aplicação de medidas corretivas e/ou preventivas são determinadas e aplicadas assim que a causa raiz do problema é identificada com o intuito de evitar que problemas recorrentes ocorram no futuro.

\section{CONCLUSÃO}

Este trabalho teve como propósito analisar a relação entre o uso da metodologia lean e o desempenho de prazo de projetos em Organizações Representativas de Pesquisa Clínica.

Os resultados da pesquisa permitiram constatar que mesmo em estágios iniciais de implantação da metodologia lean como novo modelo de negócio da empresa, a inclusão dos princípios como parte da missão, cultura e valores da companhia bem como a inclusão das ferramentas como parte dos processos operacionais podem contribuir com a entrega antecipada das atividades dos projetos e facilitar a obtenção dos objetivos estratégicos da empresa.

A presente pesquisa também comprova que a filosofia de administração de produção que teve início no Japão também pode ser aplicada na área de saúde. Na empresa estudada, os benefícios da implementação da metodologia lean em diferentes níveis organizacionais sugerem aumento da satisfação dos clientes por meio da melhor utilização de recursos em todos os níveis da organização.

Adicionalmente, durante o processo de revisão de literatura, notou-se que diversos trabalhos foram publicados nas últimas décadas sobre empresas que adotaram a filosofia lean como estratégia de sobrevivência que garanta ganhos na produtividade e vantagens competitivas, porém este ainda é um fenômeno experimentado por um seleto grupo de Organizações Representativas de Pesquisa Clínica. Sendo assim, esta pesquisa também colaborou com dados complementares sobre a metodologia lean no ramo de pesquisa clínica que é um mercado ainda pouco explorado.

É importante ressaltar ainda que as informações e resultados desta pesquisa sugerem a necessidade de trabalhos futuros com foco em possíveis alternativas e soluções para gerenciamento das métricas disponíveis na empresa e também de ações complementares para aprimorar o planejamento das atividades dos projetos de estudo clínico.

Ainda é importante acrescentar que esta pesquisa apresenta limitações, uma vez que apenas quatro funcionários foram entrevistados e, por isso, é possível que os resultados contidos 
neste trabalho não reflitam a percepção da maior parte dos colaboradores. Outra limitação está intimamente relacionada ao método de pesquisa utilizado, já que o estudo de caso apenas permite retratar a realidade de uma empresa. Como sugestão para futuras pesquisas, pode-se recomendar a inclusão de funcionários de diversas partes do mundo e de diferentes unidades de negócios, a fim de obter resultados mais precisos.

\section{REFERENCIAS}

ATKINSON, R. Project management: cost, time and quality, two best guesses and a phenomenon, its time to accept other success criteria. International Journal of Project Management, v. 17, n. 6, p. 337 342, 1999.

BARBER, E. Benchmarking the management of projects: a review of current thinking. International Journal of Project Management, v. 22, p. 301-307, 2004.

BRUNET, A.P.; NEW, S. Kaizen in Japan: An Empirical study. International Journal of Operations \& Production Management, v. 23, n. 12, p. 1426-1446, 2003.

CHAN, F.T.S.; LAU, H.C.W.; IP, R.W.L.; CHAN, H.K.; KONG, S. Implementation of total productive maintenance: a case study, International Journal Production Economics, v. 95, n. 1, p. 71-94, 2005.

ATTADIA. L.; MARTINS, R. Medição de desempenho como base para evolução da melhoria contínua. Revista Produção, v. 13, n. 2, p. 33-41, 2003.

CIRIELLO, D.M. Estratégias para o gerenciamento do cronograma do projeto: Dicas úteis para os gerentes de projetos e membros da equipe. Livraria Virtual PMI. Project Management Institute, 2011. Disponível em: https://brasil.pmi.org/brazil/KnowledgeCenter/Articles/ /media/C1CEEAF8C93 64BAA9FAB0D7F2B92203F.ashx. Acesso em 07 de abril de 2016.

KERZNER, H. Project Management Best Practices. New Jersey: Jon Wiley \& Sons, $2{ }^{a}$ edição, 2010.

KHADEM, M.; ALI, S.A.; SEIFODDINI, H. Efficacy of lean metrics in evaluation the performance of manufacturing systems. International Journal of Industrial Engineering, v. 15, n. 2, p. 176-184, 2008.

Lean Institute Brasil (LIB). Disponível: 〈http://www.lean.org.br/default.aspx>. Acesso em 13 de fevereiro de 2016.

LING, F. Y. Y. How project managers can better control the performance of designbuild projects. International Journal of Project Management, v. 22, p. 477488, 2004.

MICHALSKA, J.; SZEWIECZEK, D. The 5S methodology as a tool for improving the organisation. Journal of Achievements in Materials and Manufacturing Engineering, v. 24, n. 2, p. 211-214, 2007.

NEELY, A. Measuring business performance. London: The Economist Newspaper and Profile Books, 1998.

OISEN, R.P; Can Project Management be defined? Project Management Quarterly. v.2, n.1, p. 12-14, 1971.

PATAH, L.; CARVALHO, M.M. Métodos de gestão de projetos e sucesso dos projetos: um estudo quantitativo do relacionamento entre estes conceitos. Revista de Gestão e Projetos, v. 3, n. 2, p. 178206, 2012.

Project Management Institute (PMI). Um guia do conhecimento em gerenciamento de projetos (Guia PMBOK). Editora Atlanta: Global Standard, 5 a edição, 2013.

ROCHA, A.V.; COSTA, F.S.; NOGUEIRA, J.F., BELMIRA, T.R. Gerenciamento de Qualidade em Projetos. Rio de Janeiro: Editora FGV, 2014. 
SILVA, L.M. A importância do gerenciamento do tempo nos projetos. Techoje. Belo Horizonte, 2016. Disponível em: http://www.techoje.com.br/site/techoje/categoria/detalhe_artigo/409.

TEICH, S. T.; FADDOUL, F. F. Lean Management - The Journey from Toyota to healthcare. Rambam Maimonides Medical Journal, v.4, n.3, p. e0007, 2013.

TOUSSAINT, M. D.; BERRY, L. L. The Promise of Lean in Healthcare. Mayo Clinic Proceedings, v.88, n. 1, p. 74-82, 2013.

WOMACK, J. P.; JONES, D. T.; ROOS, D. A máquina que mudou o mundo. Rio de Janeiro: Editora Campus, 1992.

WOMACK, J.P.; JONES, D.T. A mentalidade enxuta nas empresas - Elimine o desperdício e crie riquezas. Rio de Janeiro: Editora Campus, 1998.

ZIDEL, T.G. A Lean Guide to Transforming Healthcare: How to Implement Lean Principles in Hospitals, Medical Offices, Clinics, and Other Healthcare Organizations. Milwaukee: ASQ Quality Press, 2006.

ZU, X.; ROBBINS, T.L.; FREDENDALL, L.D. Mapping the critical links between organizational culture and TQM/Six Sigma practices. International Journal of Production Economics, v.123, n. 1, p. 86-106, 2010. 